\title{
Rotordynamic Fluid Force Moments on an Open-type Centrifugal Compressor Impeller in Precessing Motion
}

\author{
YOSHIKI YOSHIDA ${ }^{\mathrm{a}, *}$, YOSHINOBU TSUJIMOTO $^{\mathrm{a}}$, DAIZO YOKOYAMA ${ }^{\mathrm{a}}$, \\ HIDEO OHASHI $^{\mathrm{b}}$ and FUMITAKA KANO ${ }^{\mathrm{c}}$ \\ ${ }^{a}$ Osaka University, Engineering Science, 1-3, Machikaneyama, Toyonaka, Osaka 560-8531, Japan, \\ ${ }^{\mathrm{b}}$ Kougakuin University, 1-24-2, Nishishijuku, Shinjuku, Tokyo 163-8677, Japan; \\ ${ }^{\mathrm{c}}$ Nara National College of Technology, 22, Yata, Yamatokoriyama, Nara 639-1058, Japan
}

(Received 15 May 2000; In final form 23 May 2000)

In recent years, increasing interest has been given to the rotordynamic fluid forces on impellers, from the view point of the shaft vibration analysis. Previous experimental and analytical results have shown that the fluid-induced forces and moments on closedtype pump impellers contribute substantially to the potential destabilization of subsynchronous shaft vibrations. However, to date few papers are known of the rotordynamic fluid forces on open - type centrifugal impellers. This paper reports about experimental investigations of the rotordynamic fluid force moments on an open-type centrifugal compressor impeller in precessing motion. For open-type impellers, the variations of the tip clearance and the clearance between the back shroud and casing due to the precessing motion contribute to the rotordynamic fluid force moments. Experiments were conducted to measure the rotordynamic fluid force moments directly using the 4-axis sensor, and the unsteady pressure on the front and back casing wall.

In this paper, following results are obtained: (1) The fluid force moment becomes destabilizing in the region of negative precessing speed ratio $(-0.3<\Omega / \omega<0)$, at the design flow rate; (2) At reduced flow rate, the destabilizing fluid force moments occurred at small positive precessing speed ratio $(0.2<\Omega / \omega<0.4)$; (3) From the comparison of direct measured fluid force moments with those estimated from the unsteady pressure measured on the front and back casing walls, it was found that the destabilizing moments in the backward precession are mainly caused by the fluid forces on the front surface of the present impeller, where there is large clearance between the back shroud and casing.

Keywords: Rotordynamic fluid force moment; Centrifugal impeller; Precessing motion; Selfexcited vibration; Unsteady pressure; Blade force

\footnotetext{
*Corresponding author. e-mail: yoshida@me.es.osaka-u.ac.jp
} 


\section{INTRODUCTION}

For turbomachinery operating at supercritical shaft speed it is important to understand the characters of rotordynamic fluid forces and moments on impeller which occur in response to shaft vibration. Figure 1(a) shows the typical vibration of overhung impeller. The motion of impeller can be divided into two fundamental modes (Ohashi et al., 1991). One is whirling motion, and the other is precessing motion. The vibration of a rotating impeller (rotational speed $\omega$ ) can be dissolved into two modes; whirling with a constant eccentricity, $\varepsilon$, as shown in Figure 1(b), and precession with a

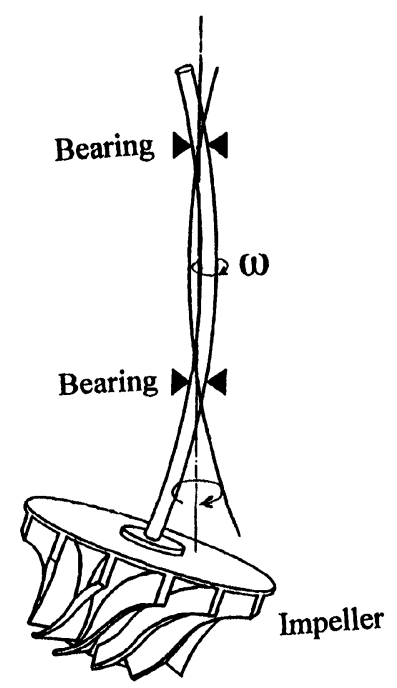

(a) Vibrating motion of a overhung impeller

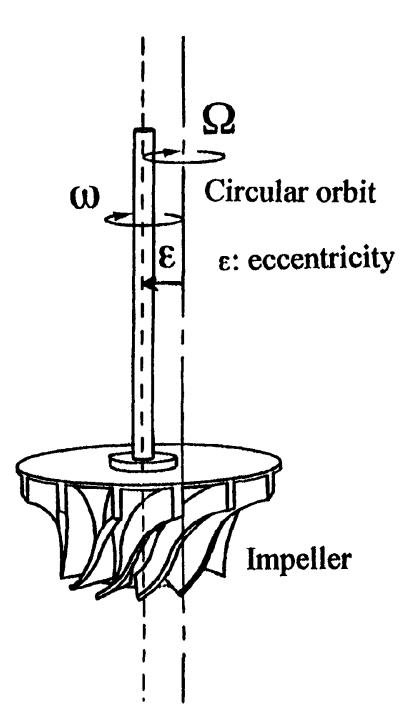

(b) Whirling motion

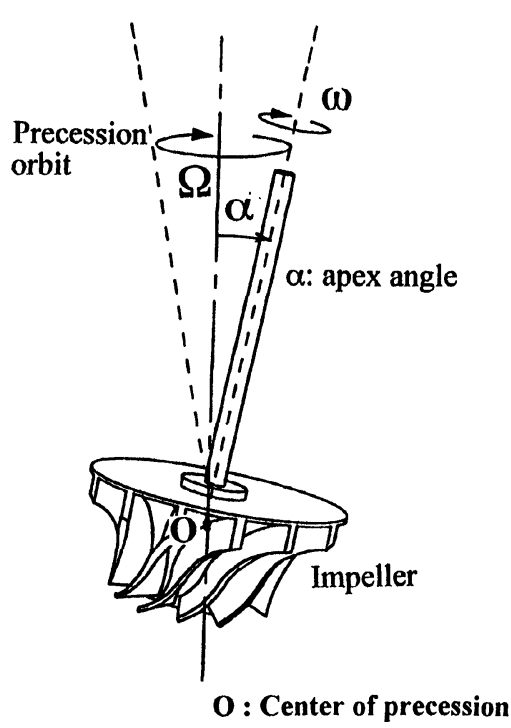

(c) Precessing motion

FIGURE 1 Motion of a overhung impeller on a vibrating shaft, and two fundamental vibration modes of an impeller. 
constant shaft inclination, $\alpha$, as shown in Figure 1(c). In the precession, the center of the impeller, " $O$ ", does not move but the direction of the shaft inclination rotates with constant speed $\Omega$.

For whirling motion, many experimental and analytical data have been obtained on the rotordynamic fluid forces on pump impellers, mainly at Caltech and the University of Tokyo. It is now widely recognized that for closed - type centrifugal impellers the fluid forces become destabilizing for the forward whirl generally at whirl speed ratio $(\Omega /$ $\omega$ ) less than 0.5 (Jery et al., 1985, Bolleter et al., 1987, and Ohashi et al., 1988). The destabilizing forces are caused by the unsteady interaction between the impeller and volute casing (Adkins et al., 1988, and Tsujimoto et al., 1988A) or vaned diffuser (Tsujimoto et al., 1988B), or by unsteady leakage flow between impeller shroud and casing (Childs, 1989, and Guinzburg et al., 1994). The results are summarized in the recent textbooks of Childs (1993) and Brennen (1994).

For precessing motion, Ohashi et al. (1991) have shown that, from direct force measurements on a closed-type pump impeller, the fluid force moments become destabilizing for the forward precessing speed ratio $(\Omega / \omega)$ less than 0.5 . This range of destabilizing speed ratio is quite similar to that of the whirling cases as mentioned above. Yoshida et al. (1997) and Tsujimoto et al. (1997) investigated the unsteady flow in the back shroud/casing clearance of a precessing pump impeller. They also observed that the fluid force moment on the back shroud is destabilizing at small positive precessing speed ratio. In addition, the frequency range of destabilizing moment increases as the increase in the leakage flow rate, caused by the increase in the steady circumferential velocity, $V_{\theta}$, in the back shroud/casing clearance.

Open-type impellers have been widely used for high-speed and high-pressure centrifugal compressors. For open - type impellers, the variation of the tip clearance due to the shaft vibration contributes to the rotordynamic fluid forces. Nevertheless, as far as the authors are aware, no rotordynamic data for these machines are available except the recent report of Yoshida et al. (1999). Yoshida et al., reported experimentally that for an open-type centrifugal compressor impeller in whirling motion the fluid force becomes destabilizing at small positive whirl speed ratio throughout all the flow range without the interaction of a volute or vaned diffuser. In addition, the fluid forces change dramatically into destabilizing near the whirling speed ratio $\Omega / \omega=0.8$ at the low flow rate. This peak of destabilizing fluid force is caused by the strong interaction of the whirling motion with the rotating flow instability, similar to "rotating stall", with propagating speed ratio $\Omega^{\prime} / \omega=0.79$.

This paper presents the results from an investigation of the rotordynamic fluid force moments on an open-type centrifugal compressor impeller in precessing motion. For the precessing motion, the variations of the tip clearance and the clearance between the back shroud and casing contribute to the rotordynamic fluid force moments. Fluid force moments were measured directly with a force balance device. Discussions on the unsteady pressure on the front and back casing walls, and blade to blade pressure distribution are also presented.

\section{EXPERIMENTAL FACILITY}

\section{Description of Test Facility}

Figure 2 shows the sketch of the mechanism used to generate the precessing motion. The inner sleeve supports the main shaft through two eccentric inner bearings set to produce a pure precessing motion. The main shaft is driven by an AC motor with the rotational speed $(\omega)$ through a universal joint, and the outer sleeve is driven by a DC motor controlled to run at a prescribed speed $(\Omega)$. This in term is the precessing speed. The precessing angular velocity, $\Omega$, is defined as positive when it is in the same direction as the impeller rotation, $\omega$. The main shaft speed was maintained at $400 \pm 1 \mathrm{rpm}$, and the precessing speed ratio $(\Omega / \omega)$ was varied in the range from -1.4 to +1.4 . Uncertainty in the precessing speed ratio, $\Omega / \omega$, is \pm 0.002 .

Figure 3 shows the details around the impeller. The test impeller is a model of an industrial 


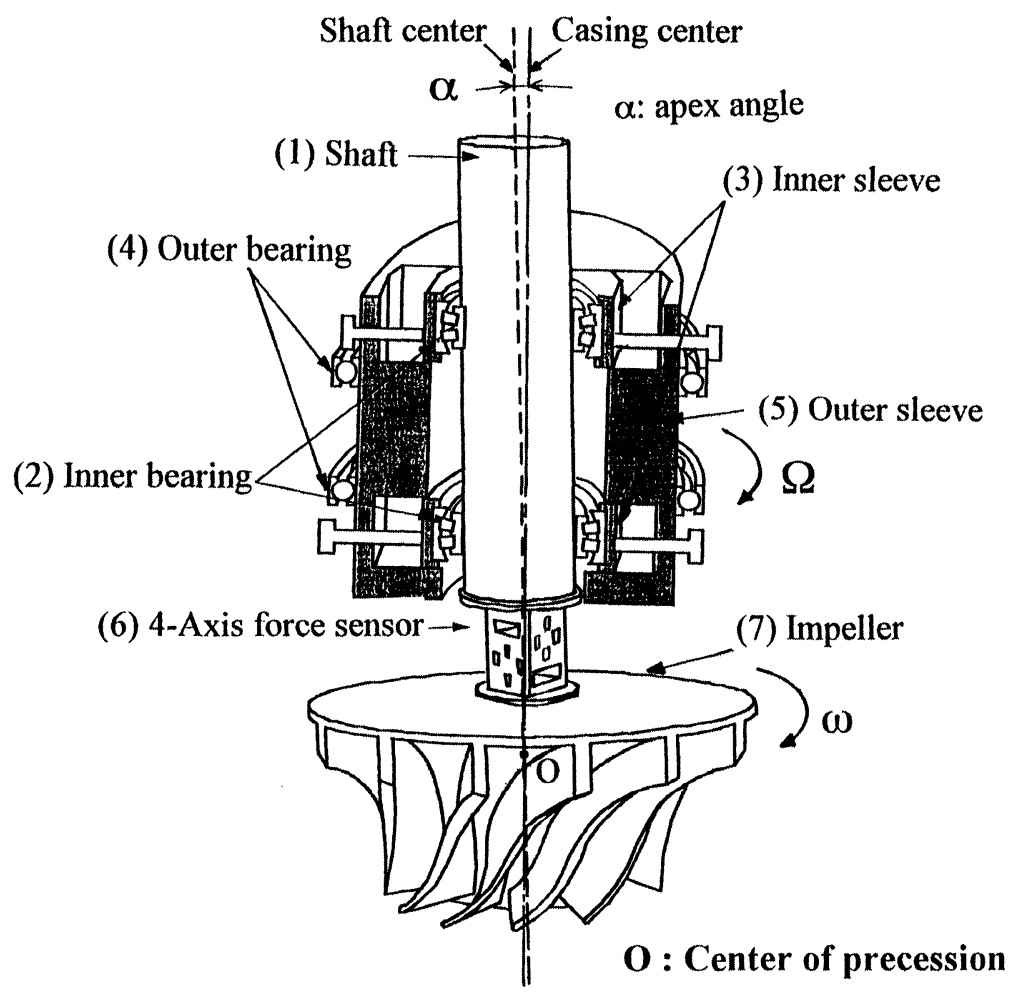

FIGURE 2 Mechanism to produce the impeller precessing motion.

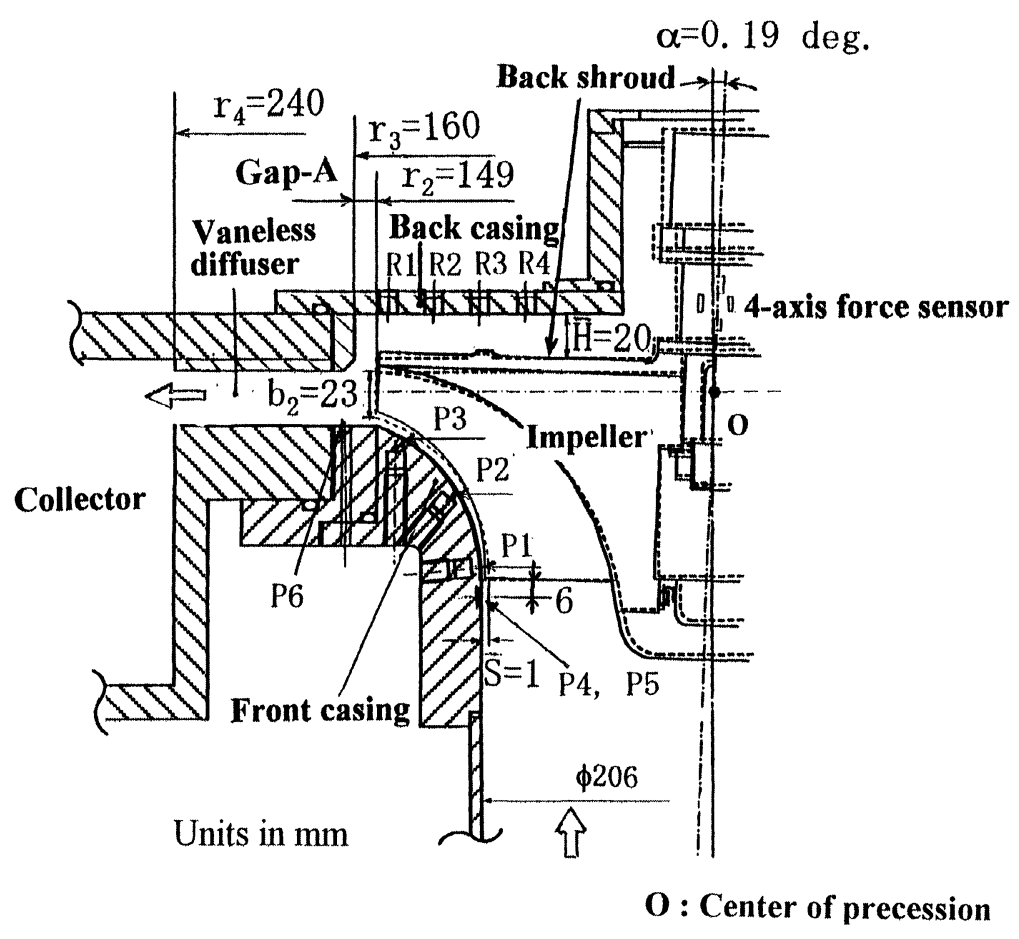

FIGURE 3 Cross section of the test rig (impeller, casing and shaft). 
centrifugal compressor with 12 blades $\left(Z_{i}\right)$, inlet blade angle $32 \mathrm{deg}$. and outlet $45 \mathrm{deg}$. at the tip (i.e., back swept blade), outer radius $\left(r_{2}\right) 149 \mathrm{~mm}$, exit width $\left(b_{2}\right) 23 \mathrm{~mm}$; its non-dimensional type number is 1.3 . The impeller is equipped with a vaneless diffuser with radius ratio $r_{4} / r_{2}=1.61$ and a symmetrical collector to minimize the nonuniformity of exiting flow. Although the test impeller was designed for gas, water was used as the working fluid to facilitate the measurement of the fluid forces. The Reynolds number $\left(\operatorname{Re}=u_{2} r_{2} / \nu\right.$, $\left.u_{2}=r_{2} \omega\right)$ is $3.0 \sim 4.0 \times 10^{6}$ for actual condition (air, $u_{2}=300 \sim 400 \mathrm{~m} / \mathrm{s}$ ), and $0.92 \times 10^{6}$ for this laboratory test condition (water, $u_{2}=6.2 \mathrm{~m} / \mathrm{s}$ ). The effect of compressibility of actual working fluid (gas) is neglected in the test condition (water). Under the condition without a shaft inclination ( $\alpha=0$ deg.), the normal blade tip clearance $(\bar{S})$ is constant $(=1 \mathrm{~mm})$ from the inlet to outlet. In the present tests, the center of precessing motion was placed at the impeller center " $O$ ", (see Fig. 3, placed on the center line of the impeller outlet height), and the apex angle $\alpha=0.19$ deg. was used for the precessing motion. In this condition, the tip clearance varies in the angular direction in the range of $0.7 \mathrm{~mm} \sim 1.3 \mathrm{~mm}$ at the impeller inlet, and $0.6 \mathrm{~mm} \sim 1.4 \mathrm{~mm}$ at the outlet due to the precessing motion. The clearance between the back shroud and casing varies in the range of $19.5 \mathrm{~mm} \sim 20.5 \mathrm{~mm}$ at the impeller outlet $\left(r=r_{2}\right)$ with the precessing motion.

In present study, we focus on the fluid force moment caused by the forces on the front surface of the impeller. For fluid force moment on the impeller back shroud, Tsujimoto et al. (1997) reported analytically and Yoshida et al. (1997) experimentally that the destabilizing moment caused by the pressure on the back shroud increases as the increase in the leakage flow through the back shroud/casing clearance due to the increase in the steady circumferential velocity, $V_{\theta}$. The magnitude of the moment increases as the decreases of the axial clearance between back shroud and casing, $\bar{H}$, and of the radial clearance between impeller side plate and casing, Gap-A (see
Fig. 3), caused by the increase in unsteady pressure fluctuation on the back shroud. From these results, the back casing of the present model compressor was designed with a wider clearance $\bar{H}$ $(=20 \mathrm{~mm})$, and a wider Gap-A $(=11 \mathrm{~mm})$ than actual ones, and with no leakage flow in the clearance to minimize the fluid force moment on the back shroud.

\section{Instrumentation and Data Acquisition System}

The impeller is supported by the main shaft through a rotating force balance with a 4-axis force sensor, as shown in Figure 2. The force balance is composed of two couples of parallel plates and 4 strain gauges per plate to measure the 4 -axis forces ( 2 forces and 2 force moments). The strain signals are taken out through a slipring. The output signals of the strain gauges are converted to two forces and two moments components using a transfer matrix determined from a dynamic calibration test. Output signals are ensembleaveraged over 32 precessing orbits based on a trigger signal that indicates the instance when both the direction of the shaft inclination and the impeller rotating angle come to a prescribed orientation. Force and force moment are measured twice, that is, in air and in water at the same rotational and precessing speed. The former measurement values merely inertia force and gyroscopic moment of the impeller itself due to the precessing motion, therefore, the fluid-induced force and force moment can be obtained by subtracting the former from the latter. In the present paper we focus only on the fluid force moments, which determines the stability for the precessing motion. Figure 4 shows the coordinate system. The $r$-axis is set in the direction of maximum blade tip clearance and the $t$-axis perpendicular to it, directed by $90 \mathrm{deg}$. from the $r$-axis in the direction of the impeller rotation. The fluid force moment $M$ is represented with its radial $\left(M_{r}\right)$ and tangential $\left(M_{t}\right)$ component. Measured fluid force moments are normalized as $\left(m_{r}, m_{t}\right)=$ $\left(M_{r}, M_{t}\right) / M_{o}, \quad$ where $\quad M_{o}=I \alpha \omega^{2}, \quad I=\rho \pi r_{2}^{2} b_{2} i^{2}$, 


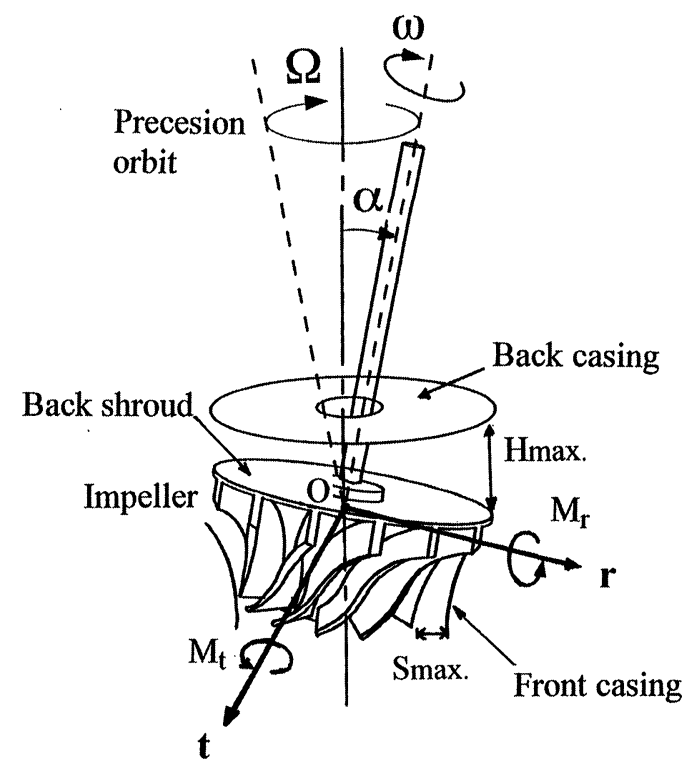

FIGURE 4 Scheme showing the rotordynamic fluid force moments. $M_{r}$ and $M_{t}$ are the radial and tangential component to the precessing motion $(\alpha$ : apex angle, $\omega$ : shaft rotational speed, $\Omega$ : precessing speed, $(r, t)$ : radial and tangential to the precessing motion).

$i=\sqrt{r_{2}^{2} / 4+b_{2}^{2} / 12}$. In the present experiment, uncertainty in the dimensionless fluid force moments $m_{r}$ and $m_{t}$ is \pm 2.0 . We should note here that the radial component of fluid force moment, $m_{r}$, is destabilizing for the precessing motion when $m_{r} \times(\Omega / \omega)>0$. (i.e., $m_{r}$ and $\Omega / \omega$ are both positive or both negative.) In this case, $m_{r} \times(\Omega / \omega)>0$, the radial moment feeds kinetic energy to the vibrating shaft and thus excitatory.

$P 1 \sim P 6$ on the front casing, and $R 1 \sim R 4$ on the back casing in Figure 3 show the locations of pressure taps to measure the steady and unsteady pressure. $P 1 \sim P 3, P 6$, and $R 1 \sim R 4$ were used to measure the steady pressure with a manometer. In addition to this, $P 1 \sim P 5$ and $R 1 \sim R 4$ were used to measure the unsteady pressure using pressure transducers to estimate the pressure force moments on the impeller (described later). $P 1 \sim P 3$ on the casing wall are facing the impeller tip, and $R 1 \sim R 4$ on the back casing wall are facing the impeller back shroud. The diameter of the pressure taps, $P 1 \sim P 3$, is $1 \mathrm{~mm}$ and silicone oil is filled in the cavity in front of the pressure transducers. The pressure transducers at $P 4, P 5$ and $R 1 \sim R 4$ were installed flush with the casing wall. $P 4$ and $P 5$ are located at different circumferencial positions (separation angle $60 \mathrm{deg}$.) to facilitate the examination of the circumferential propagation of a rotating flow instability. The resonance frequency of the measurement system is $2.2 \mathrm{kHz}$, while the blade passing frequency is $80 \mathrm{~Hz}\left(=Z_{i} \times \omega / 2 \pi\right.$. $)$.

\section{RESULTS AND DISCUSSIONS}

\section{Compressor Pressure Performance}

Figure 5 shows the static pressure coefficient $(\psi)$ at the collector and the steady pressure at various locations on (a) front casing, and (b) back casing wall plotted against the flow coefficient $(\phi)$, under the condition without the shaft inclination. The design flow coefficient is $\phi_{d}=0.424$. From the flow visualization through the transparent casing with air bubble, backflow onset at the impeller inlet was observed at $\phi=0.32$, where the pressure rise reaches a local peak. The performance curve has a positive slope in a range of $\phi=0.30 \sim 0.32$. For the measurements of fluid force moments, the flow rate was varied from $\phi=0.285$ to $\phi=0.508$. The steady circumferential velocity on the back shroud, $V_{\theta}$, was estimated from the pressure distributions in Figure 5(b). Assuming that the pressure gradient is balanced by the centrifugal force (i.e., $\left.d p / d r=\rho V_{\theta}^{2} / r=\rho K^{2} \omega^{2} r\right)$, it was found that $K=$ $V_{\theta} / r \omega$ is in the range of $0.46 \sim 0.51$ at the locations $R 1 \sim R 4$ throughout all the flow range. This result shows that the swirl on the back shroud has nearly forced vortex pattern, since there is no leakage flow in the back shroud/casing clearance.

\section{Fluid Force Moments on Impeller Measured with Force Balance}

Figure 6 shows the dimensionless radial, $m_{r}$ and tangential, $m_{t}$, fluid force moments on the impeller 


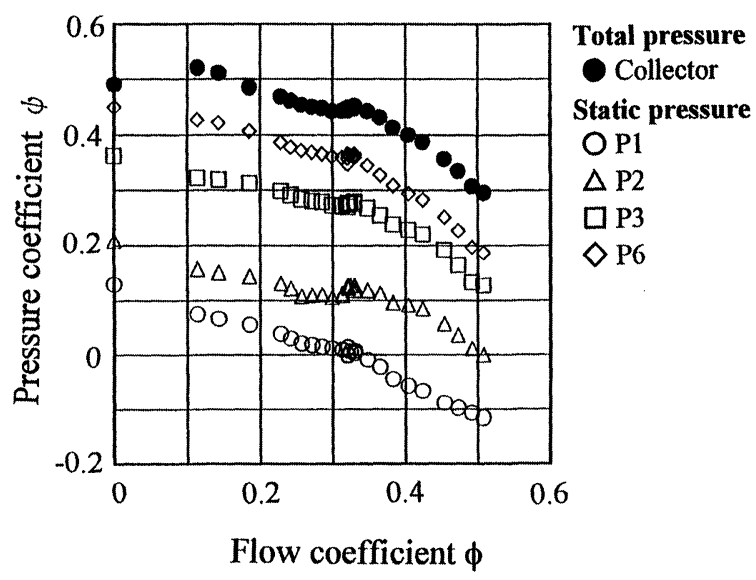

(a) Front casing (P1 P3, P6)

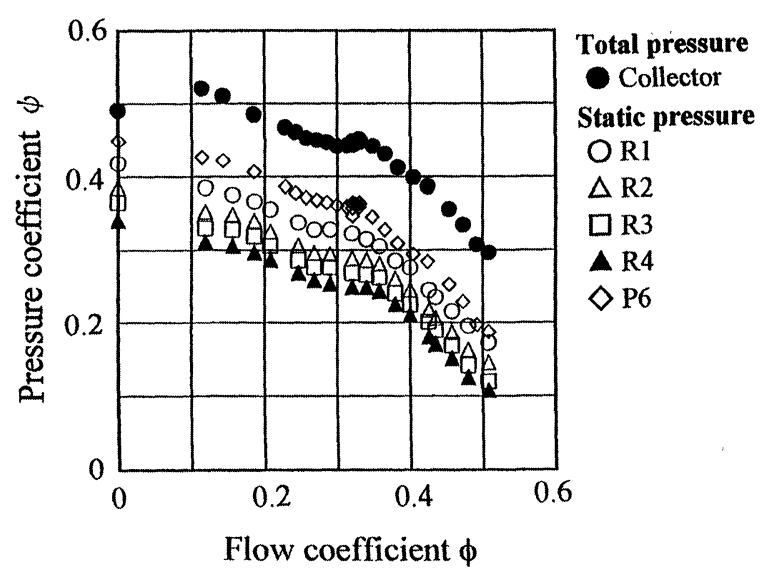

(b) Back casing (R1 R4)

FIGURE 5 Pressure performance of the test impeller and steady pressure on (a) front casing wall, and (b) back casing wall without a shaft inclination. Pressure coefficient $\psi$ versus flow coefficient $\phi$ (uncertainty in $\psi \pm 0.005$, in $\phi \pm 0.01$ ).

measured directly by the force balance versus the precessing speed ratio, $\Omega / \omega$, for various flow rates. At the design flow rate, $\phi=\phi_{d}=0.424$, it can be seen that the radial fluid force moment, $m_{r}$, on the open-type centrifugal impeller are destabilizing (i.e., $m_{r}<0$ ) for the backward precession in the range $-0.3<\Omega / \omega<0$. This destabilizing range of the fluid force moment extends to the negative direction of the precessing speed ratio as the decrease in flow rate. In addition, at lower flow rate, the radial fluid force moments become destabilizing (i.e., $m_{r}>0$ ) at positive precessing speed ratio in the range $0.2<\Omega / \omega<0.4$. Figure 7 shows the comparison of the dimensionless fluid force moments of the present open-type compressor impeller with those of the closed-type pump impeller from Ohashi et al. (1991), at the design flow rates respectively. The present opentype impeller has the destabilizing range $\left(m_{r}<0\right)$ with negative small precessing speed ratio, while the closed-type impeller has the destabilizing range $\left(m_{r}>0\right)$ with positive small speed ratio. Although the fluid force moments on the opentype impeller are smaller than those of the closedtype impeller, the radial component is roughly linear and the tangential component is quadratic with precessing speed ratio for both cases. As mentioned in the previous section, the clearance between the back shroud and casing of the present impeller, $\bar{H}=20 \mathrm{~mm}$, is wider than that of the closed-type impeller, $\bar{H}=7 \mathrm{~mm}$. This wider clearance $H$ and wider Gap-A may be one of the reasons of the smaller fluid force moments on the present open-type impeller than those on the closed-type impeller.

The peak of the force moment occurred near $\Omega /$ $\omega=0.2 \sim 0.3$ at low flow rate $\phi=0.330$. For the whirling motion of the present impeller, Yoshida et al. (1999) observed that the fluid force increases dramatically near the whirling speed ratio $\Omega / \omega=$ 0.8 at the low flow rate $(\phi<0.32)$, caused by the interaction of the whirling motion with the rotating flow instability at the impeller inlet, similar to "rotating stall", with propagating speed ratio $\Omega^{\prime} / \omega=0.79$. Childs (1989) predicted in his calculation that the peak of the force on the front shroud occurred near $\Omega / \omega=0.3 \sim 0.5$ due to the fluid structure interaction under the whirling motion. On the other hand, Tsujimoto et al. (1987) calculated the fluid forces on a whirling impeller in a vaneless diffuser using 2-dimensional vortical flow analysis. They reported that, at low flow rate, the tangential fluid force becomes destabilizing at the whirling speed ratio close to the propagating speed ratio $\Omega^{\prime} / \omega=0.157$ of the diffuser rotating stall. In the present experiment, 


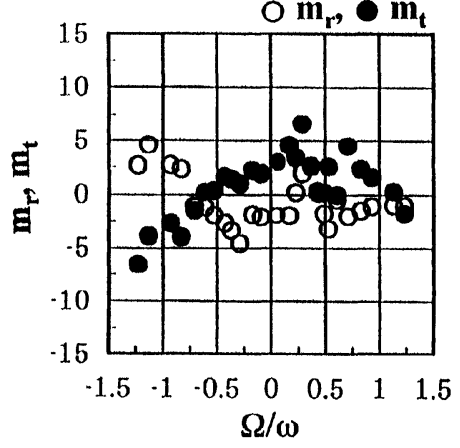

(a) $\phi=0.285$

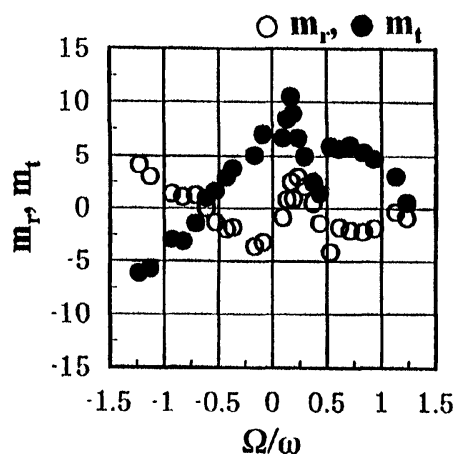

(b) $\phi=0.330$

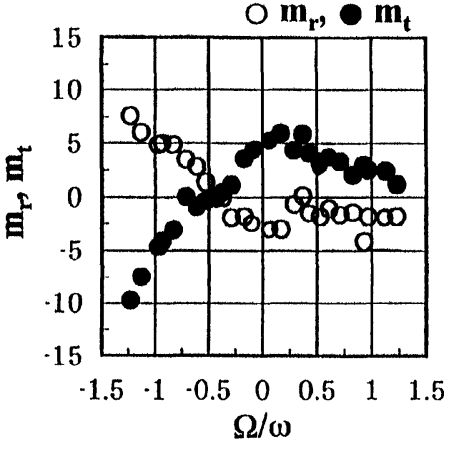

(c) $\phi=\phi_{\mathrm{d}}=0.424$

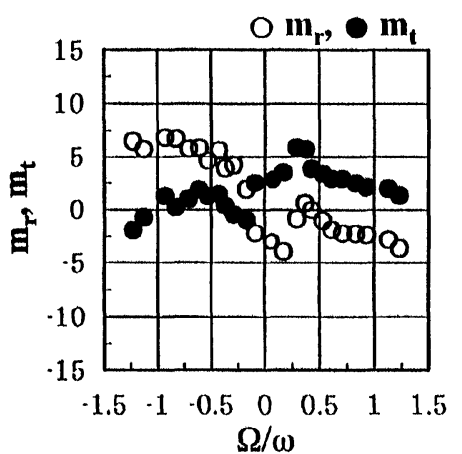

(d) $\phi=0.508$

FIGURE 6 Dimensionless fluid force moments on the impeller, radial $m_{r}$ and tangential $m_{t}$ components versus precessing speed ratio, $\Omega / \omega$, for various flow coefficients $\phi, \phi=0.285,0: 330,0.424\left(=\phi_{d}\right), 0.508$ (uncertainty in $m_{r}, m_{t} \pm 2.0$, in $\Omega / \omega \pm 0.002$, in $\phi \pm 0.01)$.

Present impeller

O 3-D closed-type pump impeller from Ohashi et al. [1991]
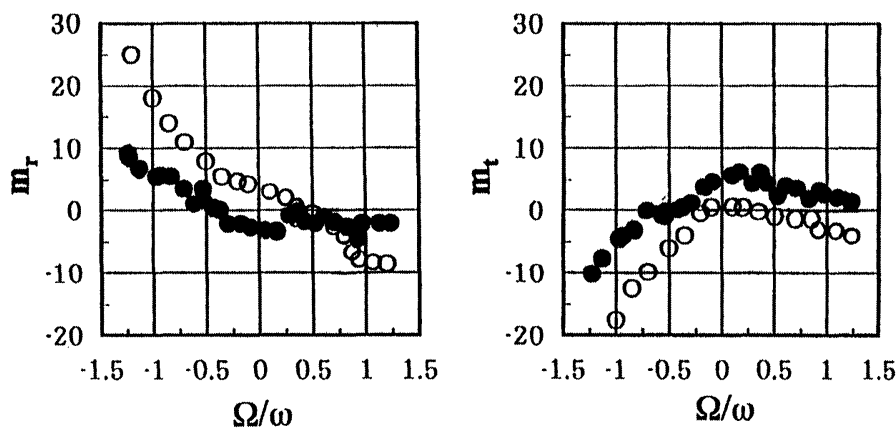

$$
\phi / \phi_{\mathrm{d}}=1.0
$$

FIGURE 7 Comparison of the dimensionless fluid force moments on the impeller, radial $m_{r}$ and tangential $m_{t}$ components, with those on the closed-type pump impeller, from Ohashi et al. (1991), at design flow rates respectively. 
we couldn't observe the rotating stall in the vaneless diffuser nor the rotating stall in the impeller inlet at this flow rate $\phi=0.330$ from the measurements of the pressure fluctuation at the diffuser inlet, $P 6$, and the impeller inlet, $P 4$ and $P 5$. Unfortunately, full explanation of this peak force moment near $\Omega / \omega=0.2 \sim 0.3$ at the low flow rate has not been obtained.

\section{Fluid Force Moments on the Back Shroud and Front Surface of Impeller}

The fluid force moments on the back shroud, and the front surface of the impeller are calculated using the direct measurements of the fluid force moment and the unsteady pressure measurements on the back casing wall to obtain a better understanding of their origin.

Unsteady pressure on the back casing wall $(\Delta p$ at locations $R 1, R 2, R 3$, and $R 4$ ) consists mainly of the precessing frequency component. Figure $8(\mathrm{a})$ shows a typical example of unsteady pressure, $\Delta p$, measured on the back casing wall at the location of $R 1$, for $\phi=\phi_{d}=0.424$, and $\Omega / \omega=0.61$. In this figure, the horizontal axis represents the phase of the precessing during a period, in which the clearance gap is widest at $\pi$, and smallest at 0 and $2 \pi$. The pressure fluctuation with precessing frequency, denoted as $\Delta p^{\Omega}$, was obtained from the Fourier analysis of $\Delta p$, as shown in the right of Figure 8(a). The fluid force moments on the back shroud were obtained from the integration of the pressure distribution between the radii $r=149 \mathrm{~mm}$ and $60 \mathrm{~mm}$ using $\Delta p^{\Omega}$ at locations $R 1,2,3$, and $R 4$.

Figure 9 shows the comparison of the dimensionless fluid force moments on the back shroud, $m_{r}^{\prime}$ and $m_{t}^{\prime}$, obtained by integrating the pressure distribution, $\Delta p^{\Omega}$, with the total moments on the impeller, $m_{r}$ and $m_{t}$, measured directly by the force balance, at $\phi=\phi_{d}=0.424$. In this figure, theoretical moments on the back shroud (from Tsujimoto et al., 1997) are also shown. It is calculated under the conditions of no leakage flow, $K=0.5$, and no resistance at Gap-A. Under these conditions, the calculated radial component is 0 for all values of $\Omega / \omega$, and the tangential component is 0 at $\Omega / \omega=-0.5$ and +1.5 with the maximum at $\Omega / \omega=+0.5$. It can be found that the tangential fluid force moment, $m_{t}^{\prime}$, shows almost the same character as the calculated moment with precessing speed ratio. The radial fluid force moment, $m_{r}^{\prime}$, on the back shroud is nearly 0 in the range $-0.3<\Omega / \omega<0$, however the radial fluid force moment, $m_{r}$, on the impeller directly measured by the force balance becomes negative (i.e., destabilizing). Furthermore, the influence of the flow rate upon the fluid force moment on the back shroud was very small.

To obtain the understanding of the fluid force moments on the "front surface" of the impeller, $m_{r}^{\prime \prime}$ and $m_{t}^{\prime \prime}$, the fluid force moments on the back shroud, $m_{r}^{\prime}$ and $m_{t}^{\prime}$, were subtracted from the direct measured moments on the impeller, $m_{r}$ and $m_{t}$, at the same test conditions. Here, we call the surface of the impeller except the "back shroud" as the "front surface". Figure 10 shows the dimensionless fluid force moments, $m_{r}^{\prime \prime}$ and $m_{t}^{\prime \prime}$, on the front surface of the impeller estimated by the above method, versus the precessing speed ratio for various flow rates. A solid line, $m_{t \text { gyro, }}$ in Figure 10(c) shows the gyro moment of the fluid in the impeller due to the precession. This $m_{\text {tgyro }}$ is agree fairly well with the tangential moment, $m_{t}^{\prime \prime}$, so that for the design flow rate some fraction of the fluid force moment on the front surface of the impeller is caused by the inertia of the fluid in the impeller. The radial component, $m_{r}^{\prime \prime}$, on the front surface of the impeller becomes destabilizing $\left(m_{r}^{\prime \prime}<0\right)$ for the backward precession with a decrease in flow rate. This tendency is similar to the radial component, $m_{r}$, on the impeller. From these results it can be concluded that the destabilizing moment $\left(m_{r}<0\right)$ in backward precession is mainly caused by the forces on the front surface of the impeller due to the variation of the tip clearance, or the blade channel height. 


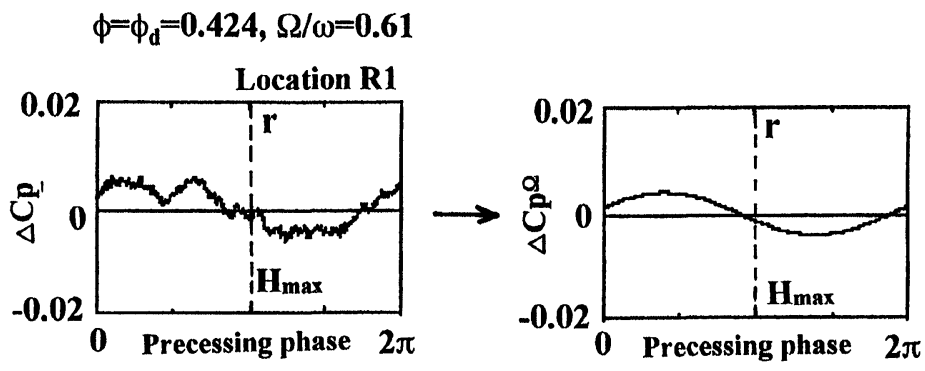

(a) $\triangle$ Cp on back casing wall

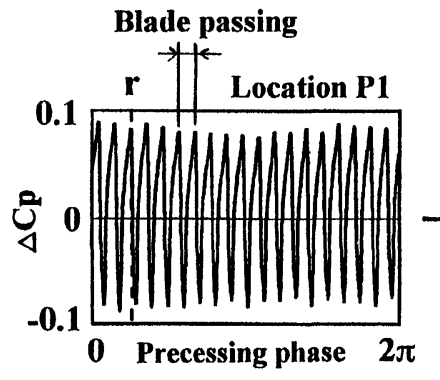

(b) $\triangle$ Cp on front casing wall
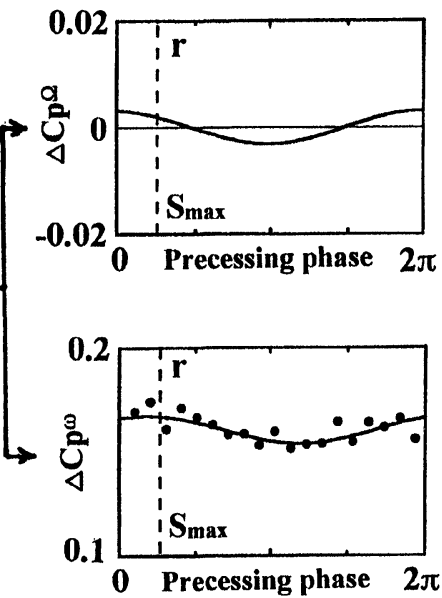

FIGURE 8 Typical wave from of unsteady pressure $\Delta C_{p}$, (a) on back casing wall at $R 1$ and (b) on front casing wall at $P 1$, for $\phi=\phi_{d}=0.424, \Omega / \omega=0.61 . \Delta C_{p}^{\Omega}$ shows the component of frequency $\Omega$, and $\Delta C_{p}^{\omega}$ shows the plot of the amplitude of the blade passing fluctuation (uncertainty in $\Delta C_{p} \pm 0.005$, in $\phi \pm 0.01$, in $\Omega / \omega= \pm 0.002$ ).
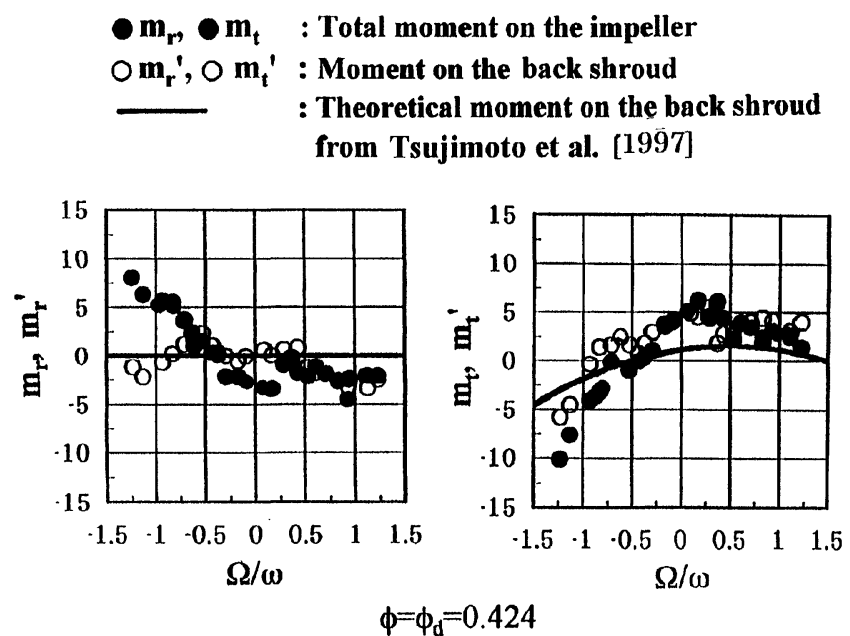

FIGURE 9 Comparison of the dimensionless fluid force moments on the back shroud, radial $m_{r}^{\prime}$ and tangential $m_{t}^{\prime}$ components, with moments on the impeller, $m_{r}$ and $m_{t}$, for design flow rate $\phi=\phi_{d}=0.424$ (uncertainty in $m_{r}, m_{t}= \pm 2.0$, in $\Omega / \omega= \pm 0.002$ ). 


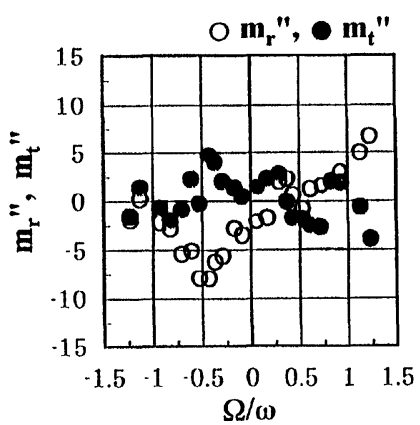

(a) $\phi=0.285$

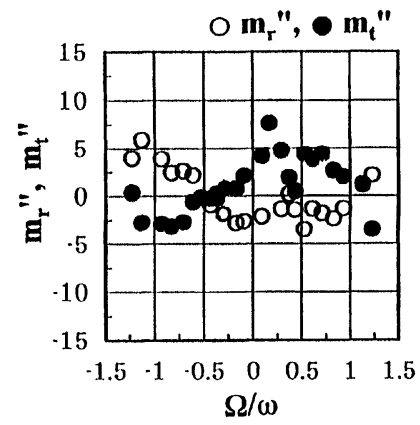

(b) $\phi=0.330$ $-\mathbf{m}_{\text {tgyro }}$

: Gyro moment on the fluid

in the impeller

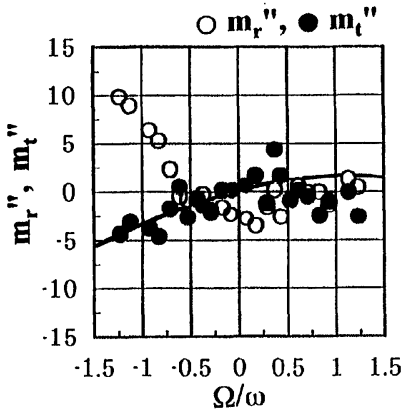

(c) $\phi=\phi_{\mathrm{d}}=0.424$

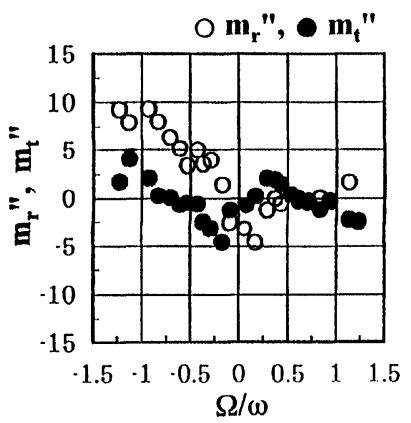

(d) $\phi=0.508$

FIGURE 10 Dimensionless fluid force moments on the front surface of the impeller, radial $m_{r}^{\prime \prime}$ and tangential $m_{t}^{\prime \prime}$ components, versus precessing speed, $\Omega / \omega$, for various flow coefficients $\phi, \phi=0.285,0.330,0.424\left(=\phi_{d}\right), 0.508$ (uncertainty in $\phi \pm 0.01$, in $\Omega / \omega= \pm 0.002)$.

\section{Force Moments on Front Surface of Impeller Estimated from Unsteady Pressure}

Two simple estimations of the force moment on the front surface of the impeller are employed. The first is the integration of the pressure distribution on the front casing wall. In this case the forces resulted from the pressure distribution and the momentum transfer at the impeller inlet and outlet, and the rate of change of fluid momentum in the impeller are neglected. The second is the integration of the blade forces evaluated from the pressure difference across the blade measured on the front casing wall. This corresponds to the nonuniform blade loading model in axial flow turbine proposed by Thomas (1958) and Alford (1965).
During one period of the precession, the number of blades passing by the pressure transducer on the front casing is $Z_{i} \times|\omega / \Omega|$. For the condition shown in Figure $8(\mathrm{~b})$, twenty waves $\left(Z_{i} \times|\omega / \Omega|=19.7\right)$ due to the blade passing are clearly observed. The amplitudes of the component with blade passing frequency, denoted by $\Delta p^{\omega}$ and used for the estimation of the blade loading, were obtained from the reading of peak-to-peak values for each blade passing, as shown in the lower right of Figure 8(b). On the other hand, the pressure fluctuation at the precessing frequency, denoted by $\Delta p^{\Omega}$ and used for the evaluation of the casing pressure force moment, was obtained from the Fourier analysis of $\Delta p$ shown in the upper right of Figure 8(b). 
For the casing pressure force moments evaluation, the fluid force moments $\left(m_{r p}^{\prime \prime}, m_{t p}^{\prime \prime}\right)$ are obtained by integrating the pressure distributions $\Delta p^{\Omega}$ from the blade leading edge to trailing edge on the casing wall. The pressure distributions are interpolated and extrapolated from $\Delta p^{\Omega}$ at $P 1$, $P 2$ and $P 3$. For the blade load evaluation, it is assumed that $\Delta p^{\omega}$ represents the pressure difference across the blade at the tip, and the pressure difference is proportional to the square of its radius from the tip to hub. The force moments $\left(m_{r b}^{\prime \prime}, m_{t b}^{\prime \prime}\right)$ are estimated by integrating the assumed pressure differences on the three segments of the blade using $\Delta p^{\omega}$ at $P 1, P 2$ and $P 3$ taking account of the back swept blade.

Figure 11 shows the comparison of the estimated force moments (a) $\left(m_{r p}^{\prime \prime}, m_{t p}^{\prime \prime}\right)$, and (b) $\left(m_{r b}^{\prime \prime}\right.$, $\left.m_{t b}^{\prime \prime}\right)$ with the moments on the front surface of the impeller $\left(m_{r}^{\prime \prime}, m_{t}^{\prime \prime}\right)$, for the design flow rate $\left(\phi=\phi_{d}=0.424\right)$. The estimated moments ( $m_{r p}^{\prime \prime}$, $\left.m_{t p}^{\prime \prime}\right)$ and $\left(m_{r b}^{\prime \prime}, m_{t b}^{\prime \prime}\right)$ are not in good agreement with $\left(m_{r}^{\prime \prime}, m_{t}^{\prime \prime}\right)$. Thomas (1958) and Alford (1965) explained the destabilizing mechanism in axial flow turbines from the blade loading nonuniformity due to the change in tip clearance. Yoshida et al. (1999) reported that for the whirling opentype centrifugal impeller some fraction of the fluid force on the impeller is caused by the nonuniform pressure distribution on the casing wall. However, the present results suggest that the fluid force moment on the front surface of the tested impeller cannot be approximated by the nonuniform blade loading nor nonuniform pressure distribution on the casing wall. Therefore, a
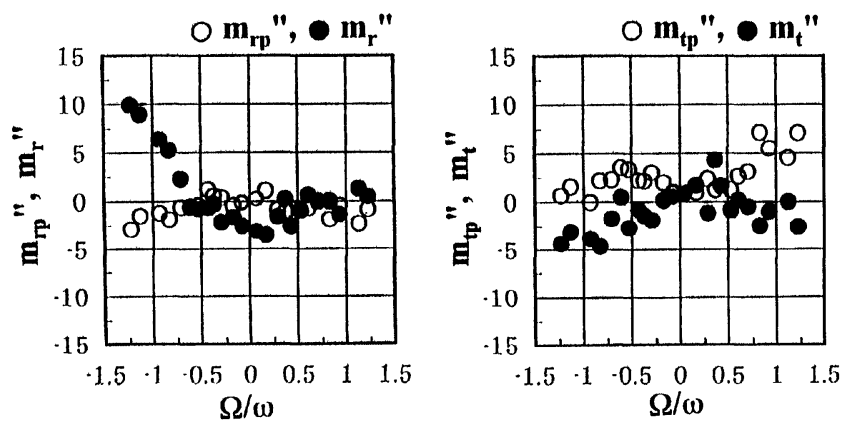

(a) $\mathbf{m}_{\mathrm{rp}} ", \mathbf{m}_{\mathrm{tp}}{ }^{\prime}\left(\phi=\phi_{\mathrm{d}}=0.424\right)$
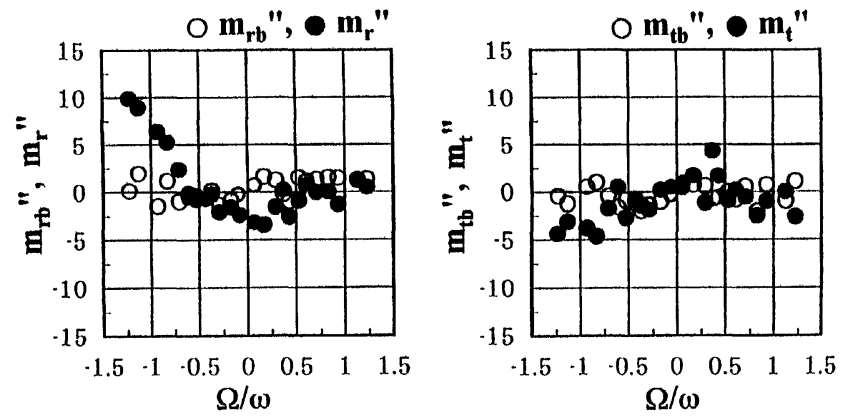

(b) $\mathbf{m}_{\mathrm{rb}} ", \mathbf{m}_{\mathbf{t b}} "\left(\phi=\phi_{\mathrm{d}}=0.424\right)$

FIGURE 11 Comparison of the dimensionless fluid force moments on the front surface of the impeller, radial $m_{r}^{\prime \prime}$ and tangential $m_{t}^{\prime \prime}$ components with (a) moments, $m_{r p}^{\prime \prime}$ and $m_{t p}^{\prime \prime}$, estimated from $\Omega$ component of unsteady pressure on the front casing, and (b) moments, $m_{r b}^{\prime \prime}$ and $m_{t b}^{\prime \prime}$, estimated from the pressure difference across the blades, for the design flow $\phi=\phi_{d}=0.424$ (uncertainty in $\phi \pm 0.01$, in $\Omega / \omega= \pm 0.002)$. 
different flow model is needed to explain the rotordynamic fluid force moment on open-type centrifugal impellers. More detailed experiments and sophisticated analyses are necessary to estimate the fluid force moment on open-type centrifugal impellers in the future.

\section{CONCLUSIONS}

From the experimental results and discussions, the following conclusions can be drawn:

(1) For an open-type centrifugal compressor impeller, the radial fluid force moment becomes destabilizing for the backward precessing motion in the range $-0.3<\Omega / \omega<0$ at the design flow rate $(\phi=0.424)$. This destabilizing range of the fluid force moment extends to the negative direction of the precessing speed ratio as the decrease in flow rate $(\phi=0.285 \sim$ 0.330 ).

(2) At lower flow rate $(\phi=0.285 \sim 0.330)$, the radial fluid force moment becomes destabilizing also for the forward precessing motion in the range $0.2<\Omega / \omega<0.4$.

(3) From the direct measured moments and the unsteady pressure measurements on the back casing, it was shown that the destabilizing fluid force moments on the impeller at negative precessing speed ratio $(-0.3<\Omega /$ $\omega<0)$ are mainly caused by the forces on the front surface of the present impeller, where there is a large clearance in the back casing wall.

(4) The force moments on the front surface of the impeller estimated from the unsteady pressure on the front casing wall, and the pressure difference across the blade don't agree with the direct measured force moments.

(5) More detailed experiments and sophisticated analyses are needed to explain the fluid force moments on open-type centrifugal impellers in precessing motion, and get more general conclusion.

\section{Acknowledgments}

The authors wish to express their gratitude for the effort of Mr. Isamu Fukushima in support of this program as an undergraduate project at Osaka University. The authors would like to thank Gas Turbine Society of Japan for permission to publish this paper that was presented in the 7th International Gas Turbine Congress 1999, Kobe. The present study was partly supported by the Ministry of Education, Science, Sports and Culture through the Grant-in Aid for Developmental Scientific Research.

\section{NOMENCLATURE}

$b_{2} \quad$ impeller axial width at outlet $=23 \mathrm{~mm}$ (see Fig. 3)

$\Delta C_{p} \quad$ coefficient of unsteady pressure $\Delta p$, normalized by $\rho\left(r_{2} \omega\right)^{2}$

$\Delta C_{p}^{\Omega} \quad$ coefficient of unsteady pressure $\Delta p^{\Omega}$, normalized by $\rho\left(r_{2} \omega\right)^{2}$

$\Delta C_{p}^{\omega} \quad$ coefficient of unsteady pressure $\Delta p^{\omega}$, normalized by $\rho\left(r_{2} \omega\right)^{2}$

$f \quad$ frequency $(\mathrm{Hz})$

Gap-A clearance between impeller side plate and casing $=11 \mathrm{~mm}$ (see Fig. 3)

$H$ clearance between back shroud and casing (normal clearance $\bar{H}=$ $20 \mathrm{~mm}$ )

I moment of inertia of a disk with radius $r_{2}$, thickness, $b_{2}=\rho \pi r_{2}^{2} b_{2} i^{2}$, where $i=\sqrt{r_{2}^{2} / 4+b_{2}^{2} / 12}$

$K \quad$ velocity ratio $\left(=V_{\theta} / r \omega\right)$

$M_{r}, M_{t}$ components of fluid force moment; radial $(r)$ and tangential $(t)$ component (see Fig. 4)

$M_{o} \quad$ reference value of moment $=I \alpha \omega^{2}$, where $I=\rho \pi r_{2}^{2} b_{2} i^{2}, i=\sqrt{r_{2}^{2} / 4+b_{2}^{2} / 12}$

$m_{r}, m_{t}$ dimensionless fluid force moment on impeller, radial $(r)$ and tangential $(t)$ components, normalized by $M_{o}$ 


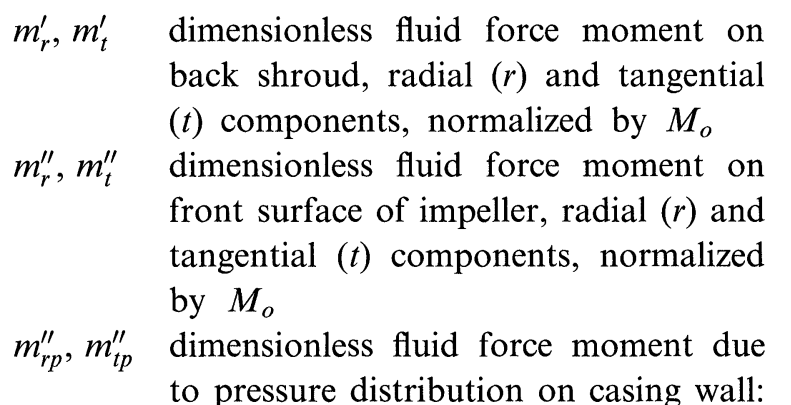
to pressure distribution on casing wall:
$\Delta p^{\Omega}$, radial $(r)$ and tangential $(t)$ components, normalized by $M_{o}$

$m_{r b}^{\prime \prime}, m_{t b}^{\prime \prime}$ dimensionless fluid force moment due to pressure difference across the blades: $\Delta p^{\omega}$, radial $(r)$ and tangential $(t)$ components, normalized by $M_{o}$

$m_{\text {tgyro }} \quad$ gyro moment of the fluid in the impeller due to the precession

p pressure

$p_{t 1} \quad$ total pressure at inlet

$\Delta p \quad$ unsteady pressure

$\Delta p^{\Omega} \quad$ amplitude of unsteady pressure on casing wall at precessing frequency

$\Delta p^{\omega} \quad$ peak-to-peak amplitude of unsteady pressure on casing wall at blade passing frequency

$r \quad$ radius

$(r, t) \quad$ radial and tangential axis (see Fig. 4)

$r_{2} \quad$ impeller outlet radius $=149 \mathrm{~mm}$ (see Fig. 3)

$S \quad$ blade tip clearance (normal clearance $\bar{S}=1 \mathrm{~mm})$

$t \quad$ time

$V_{\theta} \quad$ mean circumferential velocity in the clearance between back shroud and casing

$Z_{i} \quad$ number of impeller blades $=12$

$\alpha \quad$ apex angle of precessing motion $=0.19$ deg.

$\varepsilon \quad$ radius of circular whirl orbit

$\rho \quad$ fluid density

$\phi \quad$ flow coefficient $=$ flow rate $/\left(2 \pi r_{2}^{2} b_{2} \omega\right)$

$\psi \quad$ pressure coefficient $=\left(p-p_{t 1}\right) / \rho\left(r_{2} \omega\right)^{2}$

$\Omega \quad$ precessing angular velocity

$\Omega^{\prime} \quad$ angular velocity of rotating stall $\omega \quad$ angular velocity of impeller

$\Omega / \omega \quad$ precessing speed ratio or whirling speed ratio

$\Omega^{\prime} / \omega \quad$ propagating speed ratio of rotating stall

\section{References}

Adkins, D. R. and Brennen, C. E. (1988) "Analyses of Hydraulic Radial Forces on Centrifugal Pump Impeller", ASME Journal of Fluids Engineering, 110, 20-28.

Alford, J. S. (1965) "Protecting Turbomachinary from SelfExcited Rotor Whirl", ASME Journal of Engineering for Power, 87, 334-344.

Bolleter, U., Wyss, A., Whelte, I. and Sturchler, R. (1987) "Measurement of Hydraulic Interaction Matrices of Boiler Feed Pump Impeller", ASME Journal of Vibration, Acoustics, Stress and Reliability in Design, 109, 144-151.

Brennen, C. E. (1994) Hydrodynamics of Pumps, Concept ETI, and Oxford University Press.

Childs, D. W. (1989) "Fluid Structure Interaction Forces at Pump-Impeller-Shroud Surfaces for Rotordynamic Calculation", ASME Journal of Vibration, Acoustics, Stress and Reliability in Design, 109, 144-151.

Childs, D. W. (1993) Turbomachinery Rotordynamics, Wiley, New York.

Guinzburg, A., Brennen, C. E., Acosta, A. J. and Caughy, T. K. (1994) "Experimental Results for the Rotordynamic Characteristics of Leakage Flow in Centrifugal Pump", ASME Journal of Fluids Engineering, 116, 110-115.

Jery, B., Acosta, A. J., Brennen, C. E. and Caughy, T. K. (1985) "Forces on Centrifugal Pump Impellers", Proceedings of the 2nd International Pump Symposium, Houston, Texas, pp. $21-32$.

Ohashi, H., Sakurai, A. and Nishihama, J. (1988) "Influence of Impeller and Diffuser Geometries on the Lateral Fluid Forces of Whirling Centrifugal Impeller", NASA CP. 3026, pp. $285-306$

Ohashi, H., Imai, H. and Tsuchihashi, T. (1991) "Fluid Force and Moment on Centrifugal Impeller in Precession Motion", ASME Fluid Machinery Forum, FED, 119, $57-60$.

Thomas, H. J. (1958) "Instabile Eigenschwingungen von Turbinenlaeufern Angefacht durch die Spaltstroemung in Stopfubuchsen und Bechauchflug (Unstable Nature Vibrations of Turbine Rotors Induced by the Clearance Flows in Glands and Blading)", Bulletin de L.A.I.M., 71(11/12), 1039-1063.

Tsujimoto, Y. and Acosta, A. J. (1987) "Theoretical Study of Impeller and/or Vaneless Diffuser Attributed Rotating Stall and Their Effects on Whirling Instability of Centrifugal Impeller", Work Group on the Behavior of Hydraulic Machinery under Steady Oscillatory Conditions, Lille, France.

Tsujimoto, Y., Acosta, A. J. and Brennen, C. E. (1988A) “Theoretical Study of Fluid Forces on Centrifugal Pump Impeller Rotating and Whirling in a Volute", ASME Journal of Vibration, Acoustics, Stress and Reliability in Design, 110, $263-269$. 
Tsujimoto, Y., Acosta, A. J. and Yoshida, Y. (1988B) "A Theoretical Study of Fluid Forces on Centrifugal Pump Impeller Rotating and Whirling in a Vaned Diffuser", NASA CP. 3026, pp. 307-322.

Tsujimoto, Y., Yoshida, Y., Ohashi, H., Teramoto, N. and Ishizaki, S. (1997) "Fluid Moment on a Centrifugal Impeller Shroud in Precessing Motion", ASME Journal of Fluids Engineering, 119, 366-371.
Yoshida, Y., Tsujimoto, Y., Ohashi, H., Saito, A. and Ishizaki, S. (1997) "Measurements of the flow in Backshroud/Casing Clearance of Precessing Centrifugal Impeller", International Journal of Rotating Machinery, 3(4), 259-568.

Yoshida, Y., Tsujimoto, Y., Ishi, N., Ohashi, H. and Kano, F. (1999) "The Rotordynamic Forces on an Open-type Centrifugal Compressor Impeller in Whirling Motion", ASME Journal of Fluids Engineering, 121, 259-265. 

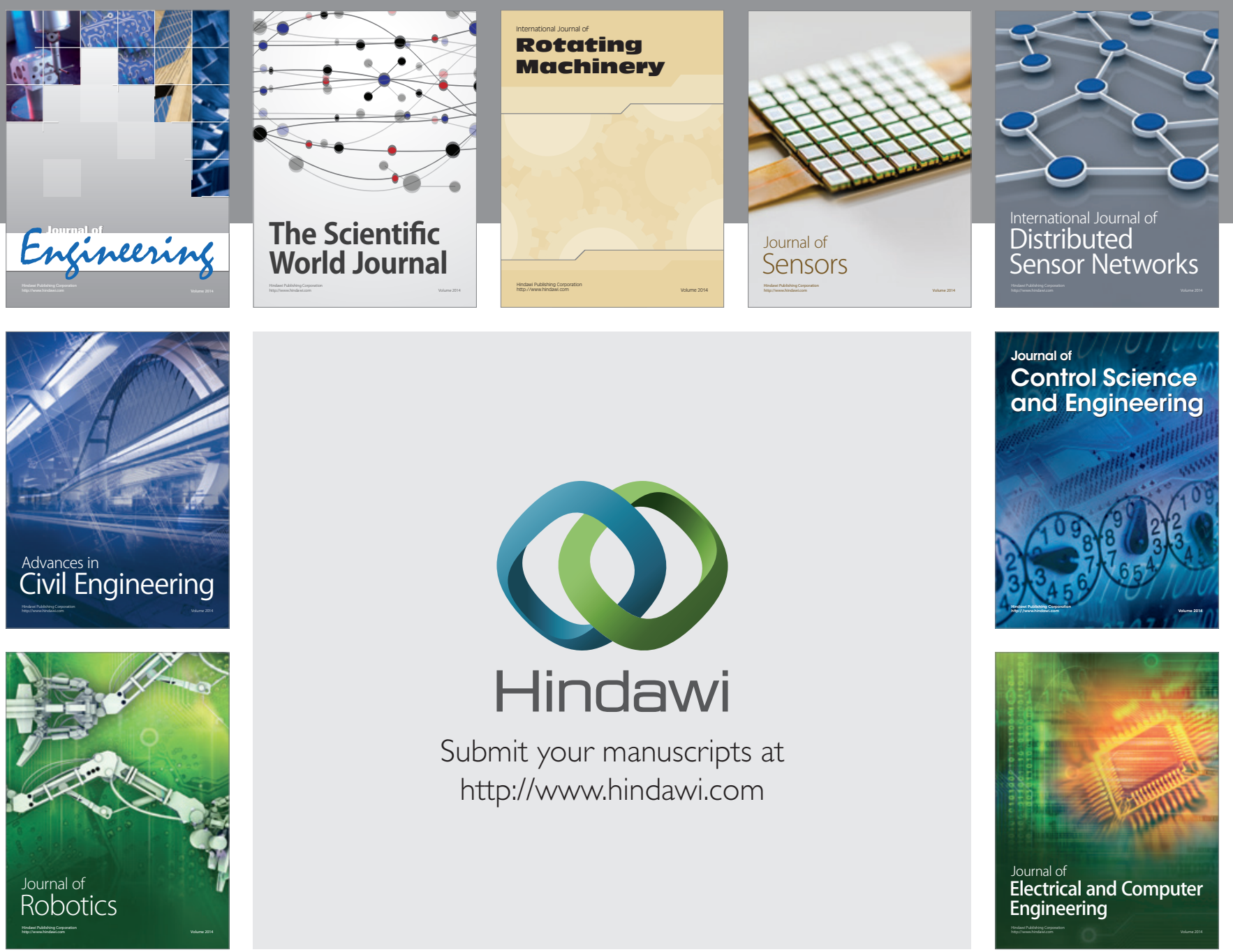

Submit your manuscripts at

http://www.hindawi.com
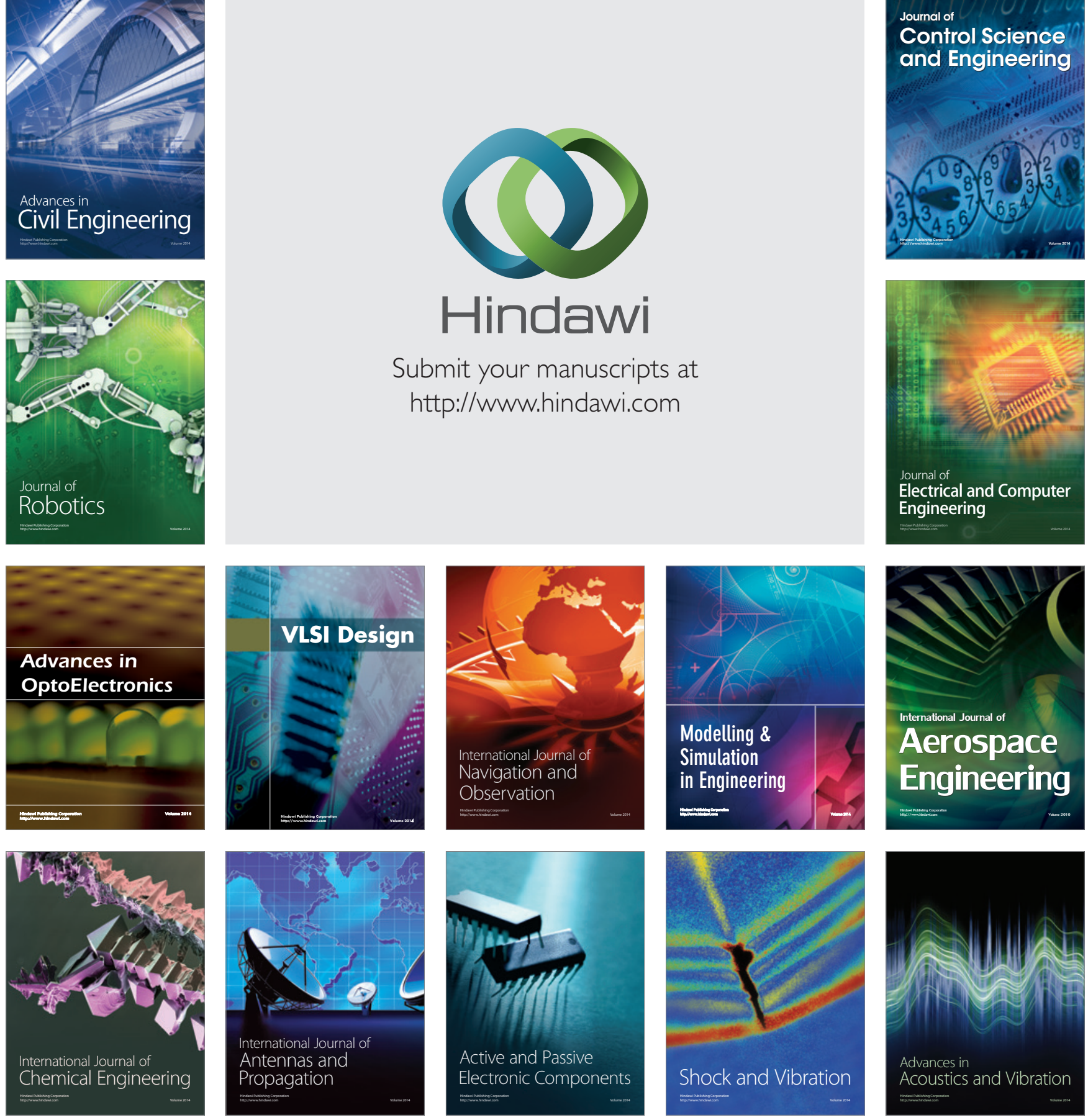